\title{
Solvability Sets in Pursuit Problem with Two Pursuers and One Evader
}

\author{
S.S. Kumkov* S. Le Ménec ${ }^{* *}$ V.S. Patsko* \\ * Institute of Mathematics and Mechanics, \\ Ural Branch of Russian Academy of Sciences; \\ Ural Federal University, Ekaterinburg, Russia \\ (e-mail: \{kumkov.jr,patsko\}@imm.uran.ru). \\ ** EADS / MBDA, Paris, France \\ (e-mail: stephane.le-menec@mbda-systems.com).
}

\begin{abstract}
The paper deals with a differential game with two pursuers and one evader. Dynamics of each object is described by a stationary linear system of a general type with a scalar control. The payoff is the minimum of two one-dimensional misses between the first pursuer and the evader and between the second pursuer and the evader. The misses are calculated at the instants fixed in advance. A method is described for constructing the level sets of the value function (i.e., the solvability ones for the game under consideration). For the case of "strong" pursuers, the optimal strategies are described. Results of simulations are given. The zero-sum game investigated can be useful for the research of the final stage of a space pursuit, where two pursuing objects and one evader are involved.
\end{abstract}

Keywords: group differential pursuit-evasion games, linear dynamics, value function, optimal feedback control, fixed termination instant

\section{INTRODUCTION}

In this paper, the authors continue study of zero-sum linear differential game with two pursuers $P_{1}, P_{2}$ and one evader $E$, which investigation have been started in works (Le Ménec (2011); Ganebny et al. (2012a,b)). All three objects move along the straight line $O z$; symbols $z_{P_{1}}, z_{P_{2}}$, and $z_{E}$ denote the coordinates of the objects on the line. At the instant $T_{1}$ prescribed in advance, the distance $\left|z_{P_{1}}\left(T_{1}\right)-z_{E}\left(T_{1}\right)\right|$ is measured between the pursuer $P_{1}$ and the evader $E$, and, at the instant $T_{2}$, the distance $\left|z_{P_{2}}\left(T_{2}\right)-z_{E}\left(T_{2}\right)\right|$ between the pursuer $P_{2}$ and the evader $E$ is measured. The payoff $\varphi$ in the game is the minimum of these two distances:

$$
\varphi=\min \left\{\left|z_{P_{1}}\left(T_{1}\right)-z_{E}\left(T_{1}\right)\right|,\left|z_{P_{2}}\left(T_{2}\right)-z_{E}\left(T_{2}\right)\right|\right\} .
$$

The first player gathering two pursuers minimizes the value of the payoff. The second player identified with the evader maximizes it.

The following description of the objects' dynamics was considered in (Le Ménec (2011); Ganebny et al. (2012a,b)):

$$
\begin{array}{ll}
\ddot{z}_{P_{i}}=a_{P_{i}}, & \ddot{z}_{E}=a_{E}, \\
\dot{a}_{P_{i}}=\left(u_{i}-a_{P_{i}}\right) / l_{P_{i}}, & \dot{a}_{E}=\left(v-a_{E}\right) / l_{E}, \\
\left|u_{i}\right| \leq \mu_{i}, & |v| \leq \nu, \\
a_{P_{i}}\left(t_{0}\right)=0, i=1,2 ; & a_{E}\left(t_{0}\right)=0 .
\end{array}
$$

The time constants $l_{P_{1}}, l_{P_{2}}$, and $l_{E}$ show how the control actions $u_{1}, u_{2}$, and $v$ act onto the accelerations created.

Differential games with dynamics (2) and the payoff function (1) having two pursuers and one evader can be interpreted (Le Ménec (2011)) as the simplest model formulations appearing in investigation of nonlinear pursuit problems in the upper atmosphere. Under this, the instant
$T_{1}\left(T_{2}\right)$ is regarded as an encounter instant on the nominal motions of the pursuer $P_{1} \quad\left(P_{2}\right)$ and the evader $E$.

In the literature devoted to one-to-one (in the sequel, $1 \times 1$ ) pursuit problems, other variants of objects with linear dynamics are also considered. For example, the paper (Shinar et al. (2013)) investigates problems with the following pursuer's dynamics:

$$
\begin{aligned}
& \ddot{z}_{P}=a_{P}, \\
& \ddot{a}_{P}=-\omega^{2} a_{P}-\zeta \dot{a}_{P}+u,|u| \leq \mu .
\end{aligned}
$$

Here, in contrast to (2), the servomechanisms' dynamics is described by a second order differential equation that corresponds to an oscillating contour with the own frequency $\omega$ and viscous friction with the factor $\zeta$.

The work (Shima $(2005))$ studied $1 \times 1$ games in the case when the control is created by deflection of aerodynamic rudders. The dynamics description is the following:

$$
\begin{aligned}
& \ddot{z}_{P}=a_{P}+d_{P} u, \\
& \dot{a}_{P}=\left(\left(1-d_{P}\right) u-a_{P}\right) / l_{P},|u| \leq \mu .
\end{aligned}
$$

The parameter $d_{P}$ is defined by the disposition of the aerodynamic rudders. Its positive (negative) values correspond to the case when the rudders are placed in the nose (tail) part of the object. As before, the symbol $l_{P}$ denotes the time constant.

Games $2 \times 1$ (two pursuers and one evader) with dynamics (3), (4) have not been studied earlier.

To make possible to take into account the dynamics variants (2)-(4), this paper considers a more general formulation, in which the linear dynamics of each object is described by its own vector differential equation with a scalar control restricted on modulus. For each object, 
the first coordinate of the phase vector is regarded as the coordinate of the object position on the straight line. It allows one to consider the payoff function in form (1).

The paper has the following structure. Section 2 gives the problem formulation. In Section 3, the passage to the equivalent differential game of the second order in the phase variable is implemented. Section 4 gives a brief description of the numerical procedure for approximate constructing the level sets of the value function (the solvability sets of the game). Section 5 is devoted to results of numerical construction of the solvability sets for several variants of problems with objects having dynamics of form (2)-(4). In Section 6, we analyze the form of the optimal strategy for the first player (that gathers the pursuers $P_{1}$ and $P_{2}$ ) in the case, when each of the pursuers is "stronger" (in its dynamic abilities) than the evader. A description of the second player's optimal strategy for this case is also given. Results of numerical simulation of the trajectories of the objects for the case of strong pursuers are considered in Section 7. Section 8 presents a conclusion.

\section{PROBLEM FORMULATION}

Let the motion of the pursuers $P_{1}, P_{2}$ and the evader $E$ be described in the vector form as follows:

$$
\begin{aligned}
\dot{\mathbf{z}}_{P_{i}}= & A_{P_{i}} \mathbf{z}_{P_{i}}+B_{P_{i}} u_{i} \\
& \left|u_{i}\right| \leq \mu_{i}, \mathbf{z}_{P_{i}} \in R^{n_{i}}, i=1,2, \\
\dot{\mathbf{z}}_{E}= & A_{E} \mathbf{z}_{E}+B_{E} v \\
& |v| \leq \nu, \mathbf{z}_{E} \in R^{n_{E}}
\end{aligned}
$$

Here, $u_{1}, u_{2}$, and $v$ are scalar controls; $A_{P_{1}}, A_{P_{2}}$, and $A_{E}$ are square matrices; $B_{P_{1}}, B_{P_{2}}$, and $B_{E}$ are columnmatrices.

Denote by $z_{P_{i}}, i=1,2, z_{E}$ the first components of the vectors $\mathbf{z}_{P_{i}}, i=1,2$, and $\mathbf{z}_{E}$.

We fix two instants $T_{1}$ and $T_{2}$. The payoff function is introduced as (1). Consider the following zero-sum differential game: the first player using controls $u_{1}$ and $u_{2}$ minimizes the payoff $\varphi$, but the second one maximizes the payoff value by its control $v$. We assume that, during the game, both players know exact values of all phase coordinates. It is necessary to propose a method for constructing the level sets of the value function (the solvability sets) and to investigate possibility of building the players' optimal strategies.

Let us agree that the game be considered with the initial instants $t_{0} \in[\bar{t}, \widehat{T})$, where $\widehat{T}=\min \left\{T_{1}, T_{2}\right\}, \bar{t}<\widehat{T}$. For certainty, we suppose that $T_{1} \geq T_{2}$.

\section{TWO-DIMENSIONAL EQUIVALENT GAME}

We denote by $x_{i}(t), i=1,2$, the value of the difference $z_{E}-z_{P_{i}}$ that is predicted from the current instant $t$ and the current positions $\mathbf{z}_{E}(t), \mathbf{z}_{P_{i}}(t)$ to the instant $T_{i}$ under the condition that zero controls act in system (5) in the interval $\left[t, T_{i}\right]$. We have

$$
x_{i}(t)=X_{E}^{1}\left(T_{i}, t\right) \mathbf{z}_{E}(t)-X_{P_{i}}^{1}\left(T_{i}, t\right) \mathbf{z}_{P_{i}}(t), i=1,2,
$$

where the upper index 1 marks the first rows of the fundamental Cauchy matrices $X_{P_{i}}\left(T_{i}, t\right)$ and $X_{E}\left(T_{i}, t\right)$ that correspond to the matrices $A_{P_{i}}$ and $A_{E}$ and are written for the instants $T_{i}$ and $t$. Since the matrices $A_{P_{i}}$, $A_{E}$ do not depend on the time $t$, the matrices $X_{P_{i}}\left(T_{i}, t\right)$ and $X_{E}\left(T_{i}, t\right)$ depend on the difference $T_{i}-t$ only. Often, the values $x_{i}(t), i=1,2$, are called the zero-effort miss coordinates (Shinar et al. (1984)). Note that $x_{i}\left(T_{i}\right)=$ $z_{E}\left(T_{i}\right)-z_{P_{i}}\left(T_{i}\right)$.

Differentiating the values $x_{i}(t)$ by $t$, we obtain

$$
\begin{aligned}
& \dot{x}_{i}(t)=X_{E}^{1}\left(T_{i}, t\right) B_{E} v-X_{P_{i}}^{1}\left(T_{i}, t\right) B_{P_{i}} u_{i}, \\
& \left|u_{i}\right| \leq \mu_{i},|v| \leq \nu, \quad t \leq T_{i}, \quad i=1,2 .
\end{aligned}
$$

From results of the differential game theory, it follows (see, for instance, (Krasovskii and Subbotin (1974, 1988); Bryson and Ho (1975))) that the differential game with dynamics (7) and the payoff function

$$
\varphi=\min \left\{\left|x_{1}\left(T_{1}\right)\right|,\left|x_{2}\left(T_{2}\right)\right|\right\}
$$

is equivalent (on value of the value function) to the differential game with dynamics (5) and payoff function (1). Computations with dynamics (7) are more convenient since the dimension of the phase vector $x=\left(x_{1}, x_{2}\right)^{\top}$ is equal two and the phase vector $x$ is absent in the right part of system (7).

Having the value $V(t, x)$ of the value function $V$ for game $(7),(8)$ at the position $(t, x)$, we obtain the corresponding equal value $\mathbf{V}(t, \mathbf{z})$ of the value function $\mathbf{V}$ in game $(5)$, (1) for any position $(t, \mathbf{z})$, where the vector $\mathbf{z}$ composed of the vectors $\mathbf{z}_{P_{1}}, \mathbf{z}_{P_{2}}, \mathbf{z}_{E}$ is such that $(t, x)$ and $(t, \mathbf{z})$ are connected by formula (6). Relation (6) is also used when we say about connection of the players' strategies in games (7), (8) and (5), (1).

Looking at system (7), note that the control $u_{i}$ affects only the variation of the coordinate $x_{i}$. But at the same time, the control $v$ determines variations of both coordinates $x_{1}, x_{2}$. Under this, if $T_{1}=T_{2}$, then in expressions for $\dot{x}_{1}(t)$ and $\dot{x}_{2}(t)$, the first summand is the same. Dynamics (7) is symmetric with respect to the origin of the plane $x_{1}, x_{2}$. The constraints on the players' control and the level sets (the Lebesgue sets) of the payoff function are also symmetric with respect to the origin. As a sequence, there exists the symmetry with respect to the origin of the time sections $W_{c}(t)$ of the level sets $W_{c}=\{(t, x): V(t, x) \leq c\}, c \geq 0$, of the value function. The set $W_{c}$ is also the solvability set corresponding to the number $c$. This is the maximal set in the space $t, x$, for which from any its point, the first player guaranties the game termination with the value of the payoff function $\varphi$ not larger than $c$. By the terminology of books (Krasovskii and Subbotin $(1974,1988))$, the set $W_{c}$ is the maximal $u$-stable bridge, corresponding to the number $c$.

Denote the scalar values $X_{E}^{1}\left(T_{i}, t\right) B_{E}$ and $X_{P_{i}}^{1}\left(T_{i}, t\right) B_{P_{i}}$ by $E_{i}(t)$ and $D_{i}(t), i=1,2$. Let

$E(t)=\left(\begin{array}{l}E_{1}(t) \\ E_{2}(t)\end{array}\right), D(t)=\left(\begin{array}{cc}-D_{1}(t) & 0 \\ 0 & -D_{2}(t)\end{array}\right), u=\left(\begin{array}{l}u_{1} \\ u_{2}\end{array}\right)$.

Then system (7) for $t \leq \widehat{T}=T_{2}$ is written in the vector form as follows:

$$
\dot{x}=D(t) u+E(t) v,\left|u_{1}\right| \leq \mu_{1},\left|u_{2}\right| \leq \mu_{2},|v| \leq v .
$$

To describe the system dynamics in the interval $\left(T_{2}, T_{1}\right]$, we consider only the first row in relation (9) for $\dot{x}_{1}$. 


\section{NUMERICAL CONSTRUCTING THE LEVEL SETS}

Efficient procedures (Isakova et al. (1984); Patsko and Turova (2001); Kumkov et al. (2005)) for backward constructing the level sets of the value function (the solvability sets) had been elaborated for linear differential games of general type with the geometric constraints $u \in \mathcal{P}$, $v \in \mathcal{Q}$ on the players' controls, a fixed terminal instant $T$, and continuous payoff function $\varphi$ that depends on two components of the phase vector at the terminal instant.

Computing two corresponding rows of the fundamental Cauchy matrix, we pass to the equivalent differential game with dynamics of type (9). After that, a partition of the interval $[\bar{t}, T]$ by instants $t_{N}=T, t_{N-1}, \ldots$ is fixed and the polygonal approximation $\widetilde{M}_{c}$ of the level set $M_{c}=$ $\{x: \varphi(x) \leq c\}$ of the payoff function $\varphi$ is chosen. (If $M_{c}$ is already a polygonal set, then it is possible to take $\left.\widetilde{M}_{c}=M_{c}\right)$. Going backward from the set $\widetilde{W}_{c}\left(t_{N}\right)=\widetilde{M}_{c}$, we build the polygonal sets $\widetilde{W}_{c}\left(t_{N-1}\right), \widetilde{W}_{c}\left(t_{N-2}\right), \ldots$ that approximate the $t$-sections $W_{c}\left(t_{N-1}\right), W_{c}\left(t_{N-2}\right), \ldots$ of the level set $W_{c}$ of the value function. Under this, we "freeze" the system dynamics in the intervals $\left[t_{k}, t_{k+1}\right)$ of the chosen partition:

$$
\dot{x}=D\left(t_{k}\right) u+E\left(t_{k}\right) v, t \in\left[t_{k}, t_{k+1}\right), u \in \widetilde{\mathcal{P}}, v \in \widetilde{\mathcal{Q}},
$$

where $\widetilde{\mathcal{P}}, \widetilde{\mathcal{Q}}$ are convex polyhedral (polygonal) approximations of the sets $\mathcal{P}, \mathcal{Q}$ of geometric constraints onto the players' controls. These sets can also be segments. The passage operation from the set $\widetilde{W}_{c}\left(t_{k+1}\right)$ to $\widetilde{W}_{c}\left(t_{k}\right)$ is chosen in such a way that the sets $\widetilde{W}_{c}\left(t_{k}\right)$ obtained approximate properly the ideal $t$-sections $W_{c}\left(t_{k}\right)$.

If the set $M_{c}$ is convex, then for any $t \leq T$ the ideal set $W_{c}(t)$ is also convex. In this case in the algorithm of the passage from $\widetilde{W}_{c}\left(t_{k+1}\right)$ to $\widetilde{W}_{c}\left(t_{k}\right)$, it is reasonable to use the formula

$$
\begin{gathered}
\widetilde{W}_{c}\left(t_{k}\right)=\left(\widetilde{W}_{c}\left(t_{k+1}\right)+\left(-\Delta t_{k}\right) D\left(t_{k+1}\right) \widetilde{\mathcal{P}}\right) * \Delta t_{k} E\left(t_{k+1}\right) \widetilde{\mathcal{Q}} \\
\Delta t_{k}=t_{k+1}-t_{k} .
\end{gathered}
$$

Here, the symbol + means the algebraic sum (the Minkowski sum), and $*$ means the geometric difference (the Minkowski difference) of two sets. Namely,

$$
\begin{gathered}
\mathcal{A}+\mathcal{B}=\{a+b: a \in \mathcal{A}, b \in \mathcal{B}\}, \\
\mathcal{A} * \mathcal{B}=\{x: x+\mathcal{B} \subset \mathcal{A}\}=\bigcap_{b \in \mathcal{B}}(\mathcal{A}-b) .
\end{gathered}
$$

If the set $M_{c}$ is not convex, but the closure of its complementation $M_{c}^{\prime}=\operatorname{cl}\left(R^{2} \backslash M_{c}\right)$ is convex, then we begin the backward constructing from the polygonal approximation of the set $M_{c}^{\prime}$. Under this, in the backward procedure, we use a formula analogous to $(10)$, but the set $\widetilde{W}_{c}^{\prime}\left(t_{k+1}\right)$ is taken as the initial one on each step; after the step, we obtain the set $\widetilde{W_{c}^{\prime}}\left(t_{k}\right)$. To get $\widetilde{W}_{c}^{\prime}\left(t_{k}\right)$, we take the set $\left(-\Delta t_{k}\right) E\left(t_{k+1}\right) \widetilde{\mathcal{Q}}$ instead of the set $\left(-\Delta t_{k}\right) D\left(t_{k+1}\right) \widetilde{\mathcal{P}}$ and the set $\Delta t_{k} D\left(t_{k+1}\right) \widetilde{\mathcal{P}}$ instead of $\Delta t_{k} E\left(t_{k+1}\right) \widetilde{\mathcal{Q}}$. The set $\widetilde{W}_{c}\left(t_{k}\right)$ is the closure of the complementation to the obtained set $\widetilde{W}_{c}^{\prime}\left(t_{k}\right): \widetilde{W}_{c}\left(t_{k}\right)=\operatorname{cl}\left(R^{2} \backslash \widetilde{W}_{c}^{\prime}\left(t_{k}\right)\right)$.

Special property of the plane is that for any polygonal set its boundary is arranged of parts with local "convexity" and "concavity", and under this, the neighboring parts have the linking edge. We use this when introduce an operator of passage from $\widetilde{W}_{c}\left(t_{k+1}\right)$ to $\widetilde{W}_{c}\left(t_{k}\right)$ in the general case when the terminal polygon $\widetilde{M}_{c}$ is not convex.

Suppose that during the backward procedure the set $\widetilde{W}_{c}\left(t_{k+1}\right)$ or $\widetilde{W}_{c}^{\prime}\left(t_{k+1}\right)$ decomposes into non-intersecting sets. (In the problem under consideration, this phenomenon takes place for $\widetilde{W}_{c}(t)$ in the case of "weak" pursuers and for $\widetilde{W}_{c}^{\prime}(t)$ in the case of "strong" pursuers.) If so, then on the next steps of the backward procedure, independent computations are performed for these sets up to their disappearing or merging.

From the point of view of numerical constructions, there are no essential complications in the case $T_{1} \neq T_{2}$. Actually, let $T_{1}>T_{2}$. Then constructing the sets $\widetilde{W}_{c}\left(t_{k}\right)$ in the interval $\left(T_{2}, T_{1}\right]$, we take into account only dynamics of the variable $x_{1}$ in system (9). Under this, the terminal set at the instant $T_{1}$ is taken in the form $M_{c}\left(T_{1}\right)=\left\{\left(x_{1}, x_{2}\right)\right.$ : $\left.\left|x_{1}\right| \leq c\right\}$. When the backward procedure comes to the instant $T_{2}$, the set $\widetilde{W}_{c}\left(T_{2}+0\right)$ obtained for this instant is united with the terminal set of the second pursuer, i.e.,

$$
\widetilde{W}_{c}\left(T_{2}\right)=\widetilde{W}_{c}\left(T_{2}+0\right) \bigcup\left\{\left(x_{1}, x_{2}\right):\left|x_{2}\right| \leq c\right\} .
$$

Further constructions are performed from this set.

\section{RESULTS OF NUMERICAL CONSTRUCTIONS}

1. For $t \in\left[\bar{t}, T_{i}\right]$, we assume

$$
\gamma_{i}(t)=\int_{t}^{T_{i}}\left(\mu_{i}\left|D_{i}(s)\right|-\nu|E(s)|\right) d s, \quad i=1,2 .
$$

The curve $t \mapsto \gamma_{i}(t), t \in\left[\bar{t}, T_{i}\right]$ going from the zero at the instant $T_{i}$ is built in the backward time $\tau_{i}=T_{i}-t$. Find the minimal number $\check{c}_{i} \geq 0$ such that $\check{c}_{i}+\gamma_{i}(t) \geq 0$ for all $t \in\left[\bar{t}, T_{i}\right]$. Then the curve $t \mapsto \check{c}_{i}+\gamma_{i}(t)$ defines the upper boundary of the solvability set $W_{\check{c}_{i}, i}$ in the individual game $P_{i}-E$. With that, $W_{\check{c}_{i}, i} \neq \varnothing$ for all $t \in\left[\bar{t}, T_{i}\right]$. The lower boundary of the set $W_{\check{c}_{i}, i}$ is defined by the graph of the function $t \mapsto-\check{c}_{i}-\gamma_{i}(t)$. If $c>\check{c}_{i}$, then the solvability set $W_{c, i}$ includes the set $W_{\check{c}_{i}, i}$. Its upper (lower) boundary differs from the upper (lower) boundary of the set $W_{\check{c}_{i}, i}$ by the shift to up (to down) by the value $c-\check{c}_{i}$. If $0 \leq c<\check{c}_{i}$, then the upper boundary of the solvability set $W_{c, i}$ is built in the backward time up to the first instant $t_{*}$ of the direct time such that $c+\gamma_{i}\left(t_{*}-0\right)<0$. With that, as above, the lower boundary is symmetric to the upper one with respect to the time axis.

2. Consider the case when the evader's behavior is described by system (2), and the behavior of each pursuer is described by system (4). Thus, if to use denotions of system (5), we have

$$
\begin{gathered}
A_{P_{i}}=\left(\begin{array}{ccc}
0 & 1 & 0 \\
0 & 0 & 1 \\
0 & 0 & -1 / l_{P_{i}}
\end{array}\right), B_{P_{i}}=\left(\begin{array}{c}
0 \\
d_{P_{i}} \\
\left(1-d_{P_{i}}\right) / l_{P_{i}}
\end{array}\right), \\
A_{E}=\left(\begin{array}{ccc}
0 & 1 & 0 \\
0 & 0 & 1 \\
0 & 0 & -1 / l_{E}
\end{array}\right), B_{E}=\left(\begin{array}{c}
0 \\
0 \\
1 / l_{E}
\end{array}\right), \\
\left|u_{i}\right| \leq \mu_{i}, i=1,2, \quad|v| \leq \nu .
\end{gathered}
$$




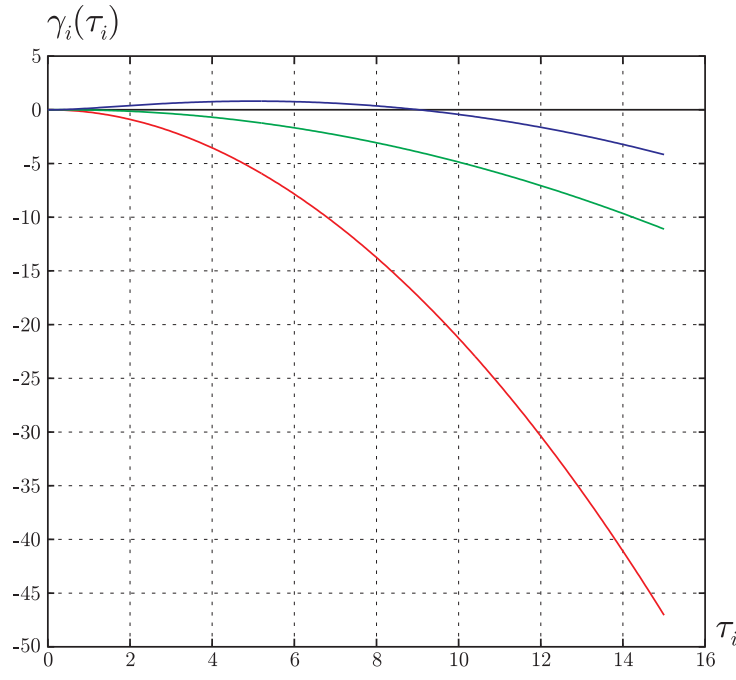

Figure 1. The curves $\gamma_{i}$ for three different variants of dynamics: $d<0$ (red line); $d=0$ (green line); $d>0$ (blue line)

Let the pursuers $P_{1}$ and $P_{2}$ be equal and $T_{1}=T_{2}=T$. Choose the values of parameters as follows: $\mu_{1}=\mu_{2}=0.9$, $\nu=1, l_{P_{1}}=l_{P_{2}}=1 / 0.9, l_{E}=1, T=15$.

Firstly, let us suppose that $d_{P_{1}}=d_{P_{2}}=d=0$. Then each of the objects has the dynamics of the first order for his control. Under this, for the chosen values of the parameters, the relations

$\frac{\mu_{i}}{\nu}=\frac{0.9}{1}<1, \frac{\mu_{i}}{\nu} \cdot \frac{l_{E}}{l_{P_{i}}}=\frac{0.9}{1} \cdot \frac{1}{1 / 0.9}=0.81<1, \quad i=1,2$,

hold. This corresponds (Shima and Shinar (2002)) to the case of weak pursuers. In the individual games $1 \times 1$ of $P_{i}$ versus $E, i=1,2$, the curve $\gamma_{i}$ that defines the kind of the solvability sets $W_{c, i}$ has the form as in Fig. 1 (the green line). The three-dimensional solvability set $W_{c}$ for $c=2.0$ in the $2 \times 1$ game is shown in Fig. 2 b. Note that $W_{0}(t)=\varnothing$ for any $t<T$ in the case of weak pursuers, i.e., there are no initial positions, from which the first player can guarantee the exact encounter.

Let now $d>0$. For $d=0.5$, the curve $\gamma_{i}$ is also shown in Fig. 1 (the blue line). The three-dimensional solvability set for $c=2.0$ is shown in Fig. 2c. It is seen that this set is significantly larger than one for $d=0$. Figure 3 presents the three-dimensional set $W_{c}$ corresponding to $c=0$. From any initial position in this set, the first player guarantees the zero miss, i.e., the exact encounter.

At last, let $d=-0.5<0$. In this case, again $W_{0}(t)=\varnothing$ for $t<T$. The curve $\gamma_{i}$ is drawn in Fig. 1 by the red line. The three-dimensional solvability set for $c=2.0$ is shown in Fig. 2a.

The sets in Fig. 2 and Fig. 3 are drawn in the same scale from the same point of view.

Thus, we can analyze and compare different variants of the solvability sets by our effective numerical algorithm.

3. Now, let us take the dynamics of form (3) for the pursuers and the dynamics of type (2) for the evader. We have
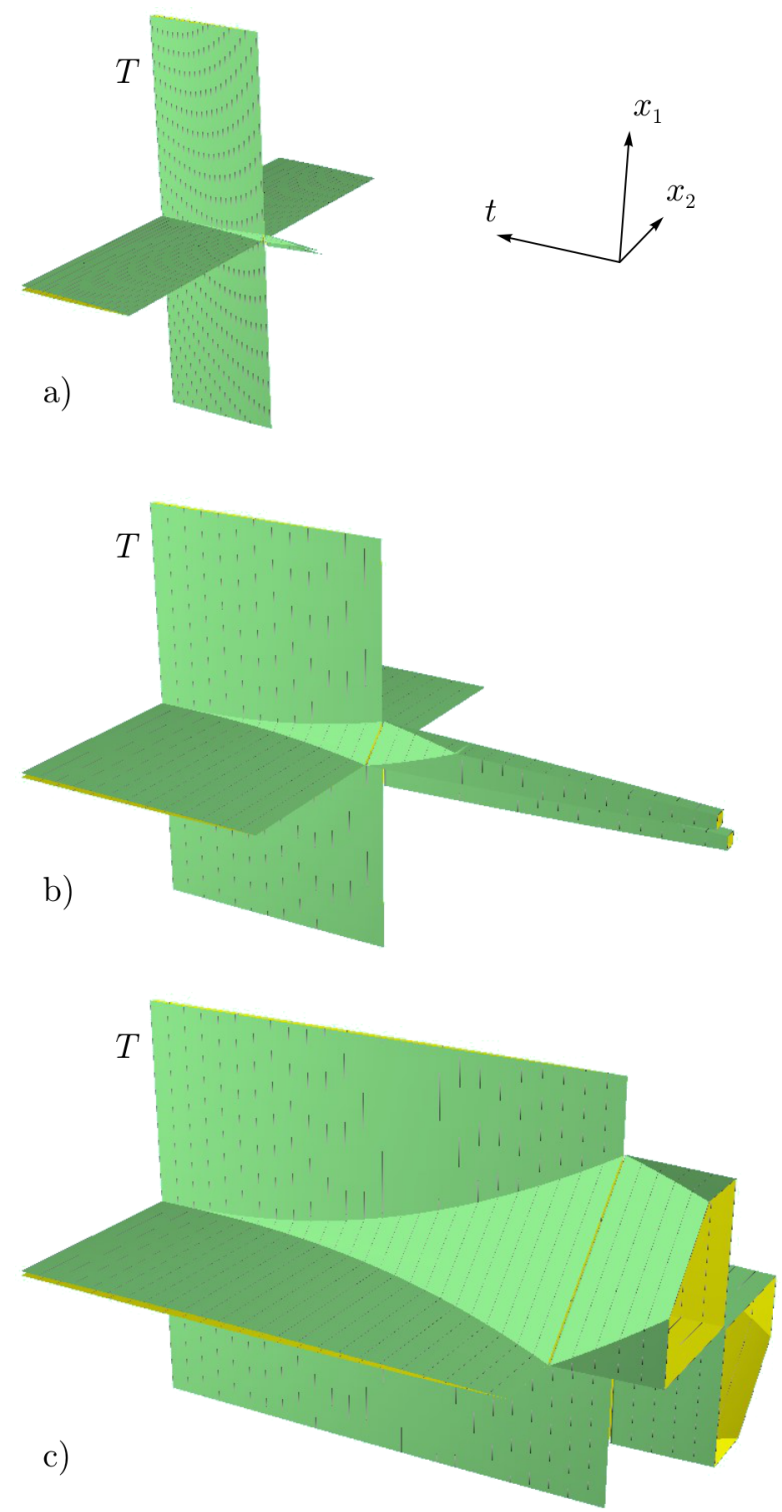

Figure 2. Solvability sets for three different variants of dynamics, $c=2.0$ : a) $d<0$, b) $d=0$, c) $d>0$

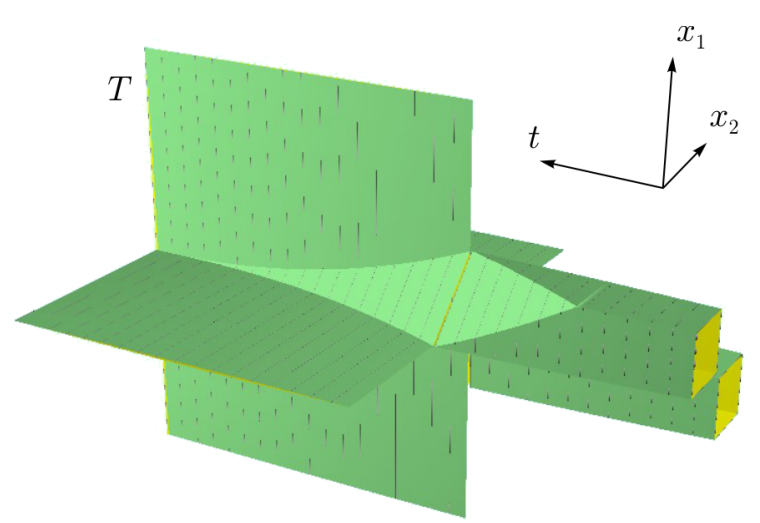

Figure 3. Capture zone (solvability set for $c=0$ ) for the case $d>0$ 


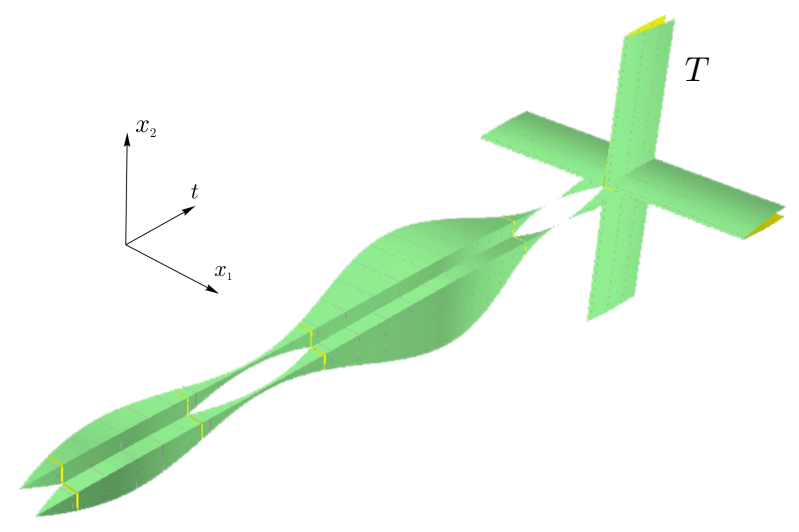

Figure 4. "Exotic" solvability set with two areas of disconnectivity of time sections

$$
\begin{gathered}
A_{P_{i}}=\left(\begin{array}{cccc}
0 & 1 & 0 & 0 \\
0 & 0 & 1 & 0 \\
0 & 0 & 0 & 1 \\
0 & 0 & -\omega_{P_{i}}^{2} & -\zeta_{P_{i}}
\end{array}\right), B_{P_{i}}=\left(\begin{array}{l}
0 \\
0 \\
0 \\
1
\end{array}\right), \\
A_{E}=\left(\begin{array}{ccc}
0 & 1 & 0 \\
0 & 0 & 1 \\
0 & 0 & -1 / l_{E}
\end{array}\right), B_{E}=\left(\begin{array}{c}
0 \\
0 \\
1 / l_{E}
\end{array}\right), \\
\left|u_{i}\right| \leq \mu_{i}, i=1,2, \quad|v| \leq \nu .
\end{gathered}
$$

Our aim is to show an example with "exotic" solvability set in a $2 \times 1$ game. Choose the following parameters: $\mu_{1}=$ $\mu_{2}=0.3, \nu=1.3, \omega_{P_{1}}=\omega_{P_{2}}=0.5, \zeta_{P_{1}}=\zeta_{P_{2}}=0.0025$, $l_{E}=1.0, T_{1}=T_{2}=30$.

Figure 4 shows the solvability set $W_{c}$ for $c=1.6$. Its "peculiarity" is in the presence of two time periods with narrow "throats". Earlier, for three-dimensional solvability sets in model problems $1 \times 1$ of cosmic pursuit, examples with one narrow throat have been constructed (Kumkov et al. (2005)). For a problem $2 \times 1$ with dynamics of form (2) in work (Kumkov et al. (2013)), an example is given, where in some period of time, the solvability set disjoins into two parts. Each of them has a narrow throat. If in a problem $2 \times 1$ the pursuers have dynamics (3), then the number of throats can be even greater. In the case of a $1 \times 1$ game of form (3) for the pursuer and of form (2) for the evader, a possibility of situation with several narrow throats have been predicted in (Shinar et al. (2013)).

\section{THE CASE OF STRONG PURSUERS}

1. We call the pursuer $P_{i}$ strong with respect to the evader $E$ if $\gamma_{i}(t)>0$ for all $t \in\left(\bar{t}, T_{i}\right)$. This means that the solvability set $W_{0, i}$ for $c=0$ in the individual game $P_{i}-E$ has nonempty non-generated $t$-sections $W_{0, i}(t)$ for all $t \in\left(\bar{t}_{i}, T_{i}\right)$.

In this section, assume that each of the pursuers is stronger than the evader.

It is evident that for $c \geq 0$ and $t \in[\bar{t}, \widehat{T}]$ the following embedding holds:

$$
\left\{x: x_{1} \in W_{c, 1}(t)\right\} \cup\left\{x: x_{2} \in W_{c, 2}(t)\right\} \subset W_{c}(t) .
$$

So, in the case of strong pursuers, the set $\operatorname{cl}\left(R^{2} \backslash W_{c}(t)\right)$ decomposes into four subsets $\mathcal{W}_{c}^{j}(t), j=\mathrm{I}$, II, III, IV, and each of them lies in its quadrant. We can consider time development of each set independently. For example, the set $\mathcal{W}_{c}^{\mathrm{I}}$ is the maximal $v$-stable bridge that terminates at $\widehat{T}$ on the convex set $\left\{x: x_{1} \geq \gamma_{1}(\widehat{T})+c, x_{2} \geq c\right\}$. For constructing the sets $\mathcal{W}_{c}^{j}$, it is possible to use the backward procedure of building the maximal $v$-stable bridge with convex $t$-sections. We can use also the theoretical results on such bridges. Particularly, since the function $V(\widehat{T}, \cdot)$ is concave on the set $\mathcal{W}_{0}^{j}(\widehat{T})$, the function $V(t, \cdot)$ for $t \in[\bar{t}, \widehat{T}]$ is also concave on the set $\mathcal{W}_{0}^{j}(t)$. For any horizontal or vertical straight line, the value $V(t, x)$ is equal to zero on the intersection of this line with the set

$$
W_{0}(t)=\operatorname{cl}\left(R^{2} \backslash \bigcup_{j} \mathcal{W}_{0}^{j}(t)\right) .
$$

The value $V(t, x)$ increases monotonically with recession of the point $x$ from this intersection segment, and further, the value becomes constant.

2. Put $u_{1}^{*}=\mu \operatorname{sign} D_{1}(t)$ at the right of the vertical axis and $u_{1}^{*}=-\mu_{1} \operatorname{sign} D_{1}(t)$ at the left. Therefore, we take the axis $x_{2}$ as the switch line for the control $u_{1}$. Similarly, take the axis $x_{1}$ as the switch line for the control $u_{2}$ : over the axis, we suppose $u_{2}^{*}=\mu_{2} \operatorname{sign} D_{2}(t)$, and below the axis, let $u_{2}^{*}=-\mu_{2} \operatorname{sign} D_{2}(t)$. At the current point $x(t)$, this method of the first player's controls provides the direction of the vector $\left(-D_{1}(t), 0\right)^{\top} u_{1}^{*}\left(\left(0,-D_{2}(t)\right)^{\top} u_{2}^{*}\right)$ to the point of minimum of the restriction of the value function $V(t, \cdot)$ onto the horizontal (vertical) line passing through the point $x(t)$. The minima of the restrictions equal zero and are reached on the axis $x_{2} \quad\left(x_{1}\right)$. Taking into account this property, it is possible to prove that the introduced very simple method of control for the first player (by the feedback principle) is optimal in the case of strong pursuers. Moreover, it is important that this method is stable with respect to small informational errors of measurement of the current state $x(t)$. For dynamics of form (2), the proof of the corresponding statement is given in (Ganebny et al. (2012a)). The optimal control synthesis of the first player is shown in Fig. 5. The light-green arrows show the direction of the vector $\left(-D_{1}(t), 0\right)^{\top} u_{1}^{*}$, and the dark-green ones correspond to the vector $\left(0,-D_{2}(t)\right)^{\top} u_{2}^{*}$.

3. In the case of strong pursuers under the additional assumption that $T_{1}=T_{2}=T$, it is easy to find also the optimal method $v^{*}$ of control for the second player.

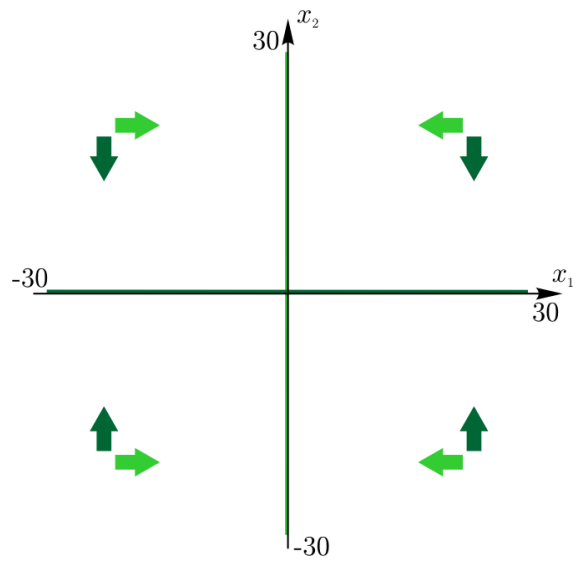

Figure 5. The optimal control synthesis of the first player 


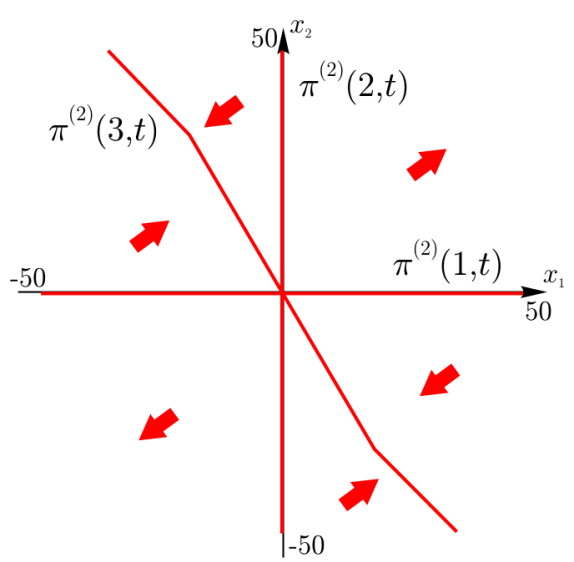

Figure 6. The optimal control synthesis of the second player

If $T_{1}=T_{2}$, then $E_{1}(t)=E_{2}(t)$. Hence, the vector $E(t)$ is directed along the bisectrix of the first and third quadrants. From this, we obtain that in the first quadrant the optimal control $v^{*}$ is calculated by formula $v^{*}=$ $\nu \operatorname{sign} E_{1}(t)$. Such a control directs the vector $E(t) v^{*}$ applied to the current point $x(t)$ to the direction of increasing the value function. In the third quadrant, the optimal control $v^{*}$ is given by the formula $v^{*}=-\nu \operatorname{sign} E_{1}(t)$. In the second quadrant for any fixed $t \in[\bar{t}, T]$, the sets $\mathcal{W}_{c}^{\mathrm{II}}(t)$ differ for any two values of $c_{1}$ and $c_{2}$ only by the shift along the bisectrix of the second quadrant. Each of the sets $\mathcal{W}_{c}^{\mathrm{II}}(t)$ has at its boundary the half-infinite horizontal ray, the half-infinite vertical ray, and the segment parallel to the bisectrix of the first and the third quadrants (parallel to the vector $E(t)$, if $E(t) \neq 0$ ) that connects the beginnings of these rays. The length of such a slant segment is the same for all $c \geq 0$ for a certain instant $t$. Let us take a ray passing through the middle points of these slant segments. We connect the beginning of the ray with the origin. In the fourth quadrant, a similar ray is parallel to the bisectrix of this quadrant. The beginning point of this ray is also joined by a segment with the origin.

On the whole, we obtain a polygonal line, which consists of two rays and the segment passing through the origin and joining the beginning points of the rays. Denote this line by $\pi^{(2)}(3, t)$ and call it the switch line for the second player. Let us take the horizontal and vertical axes as the other switch lines $\pi^{(2)}(1, t)$ and $\pi^{(2)}(2, t)$. The optimal synthesis for the second player's control at some $t$ is shown in Fig. 6 . The arrows show the direction of the vector $E(t) v^{*}$ in six cells, into which the plane $x_{1}, x_{2}$ is divided by the three switch lines. In (Ganebny et al. (2012a)) for dynamics of form (2), the statement is given on optimality of the control $v^{*}$ of the second player and its stability with respect to small errors of measurement of the position $x(t)$. The similar statement holds in the case of more general dynamics considered in this paper.

If the pursuers are strong and equal, then the switch line $\pi^{(2)}(3, t)$ does not depend on time and coincides with the bisectrix of the second and fourth quadrants.

\section{SIMULATION OF SYSTEM MOTIONS}

For representation of simulation results, consider motions of the pursuers $P_{1}, P_{2}$ and the evader $E$ in the two- dimensional plane. We call this plane the original geometric space. Assume that during the motion the horizontal component of the velocity vector of each object remains constant. Let the values of these components be such that the instants of horizontal coincidence of $P_{1}$ with $E$ and of $P_{2}$ with $E$ are the same and equal to $T$. Thus, the controls affect only onto the vertical shift. The dynamics of the lateral motion is described by relations (5); the resultant

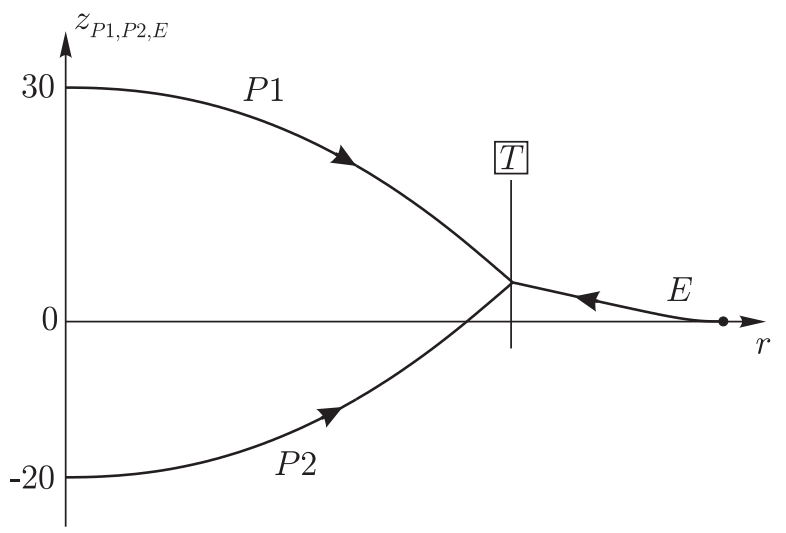

Figure 7. The trajectories of the objects in the geometric space; optimal controls of both players; the initial lateral deviations are not too large

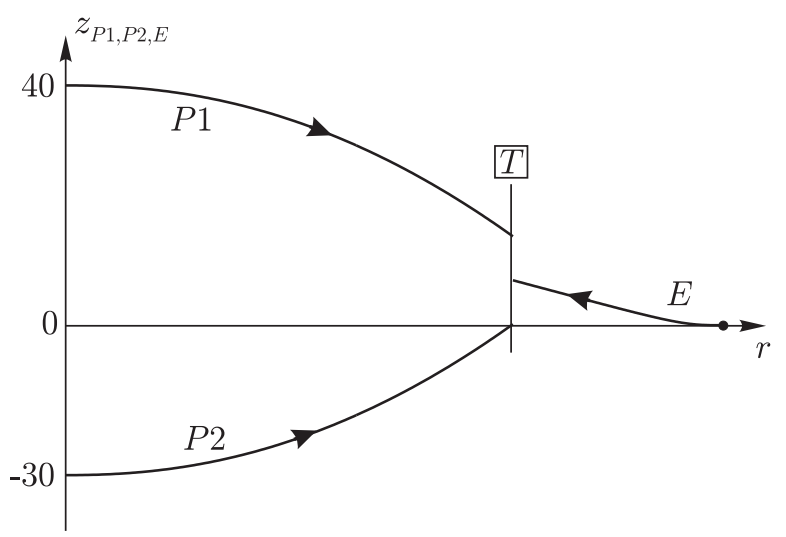

Figure 8. The trajectories of the objects in the geometric space; optimal controls of both players; the initial lateral deviations are large

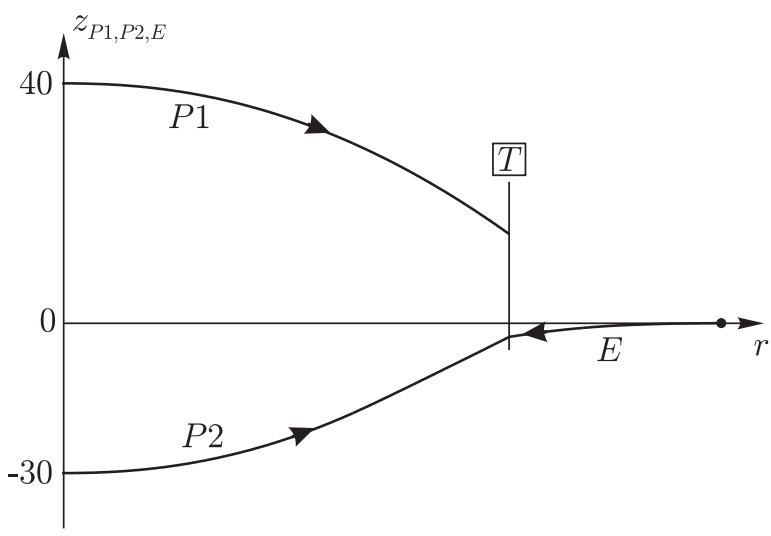

Figure 9. The trajectories of the objects in the geometric space; optimal controls of the pursuers, a random control of the evader; the initial lateral deviations are large 
miss is given by formula (1). In Figs. 7-9, the horizontal axis is denoted by the symbol $r$. So, the coordinate $r$ shows the longitudinal positions of the objects.

To simulate the trajectories, let us take a system, which solvability sets are shown in Figs. 2c and 3. The evader has a dynamics of type (2), the pursuers are equal and have their dynamics as (4).

The parameters of the game: $\mu_{1}=\mu_{2}=0.9, \nu=1$, $l_{P_{1}}=l_{P_{2}}=1 / 0.9, d_{P_{1}}=d_{P_{2}}=0.5, l_{E}=1, T_{1}=T_{2}=T=$ 8. The initial lateral velocities, accelerations and higher derivatives are zero: $\dot{z}_{P_{1}}^{0}=\dot{z}_{P_{2}}^{0}=\dot{z}_{E}^{0}=0, \ddot{z}_{P_{1}}^{0}=\ddot{z}_{P_{2}}^{0}=0$, $a_{P_{1}}^{0}=a_{P_{2}}^{0}=a_{E}^{0}=0$. The initial instant is $t_{0}=0$.

Note that the termination instant $T=8$ is taken such that both pursuers are stronger than the evader in the interval $\left[t_{0}, T\right]$ (see the blue line in Fig. 1).

Both players are controlled by the switch lines described in the previous section. Under this, they use the exact values of all phase coordinates of all objects.

In the first simulation, the initial lateral coordinates of the objects are $z_{P_{1}}=30, z_{P_{2}}=-20, z_{E}=0$. The resultant trajectories are given in Fig. 7. In this situation, there is the exact capture of the evader by both pursuers.

For the second variant, let $z_{P_{1}}=40, z_{P_{2}}=-30, z_{E}=0$ (Fig. 8). In this case, the initial lateral deviations of the pursuers from the evader are large: the pursuers are unable to provide zero payoff at the termination instant $T$.

For the third situation, we take the same initial lateral coordinates as in the second one. But now, the control of the evader is random. At the beginning of each step of the discrete scheme of control (Krasovskii and Subbotin (1974, 1988); Ganebny et al. (2012a)), it chooses randomly its control from the interval $[-\nu, \nu]$ and keeps it constant during the time step. The pursuers control optimally on the basis of the switching lines. The trajectories for some realization of the evader's control can be seen in Fig. 9. Here, the evader is captured by the second pursuer.

\section{CONCLUSION}

In the contemporary literature on the control theory devoted to pursuit problems in the upper atmosphere, different variants of dynamics for passage from the given command signal to physical acceleration of the object are described. But under this, analytical investigation of the game problems often becomes very difficult or even impossible. In the paper in application to the problem with two pursuers and one evader, the backward procedure is considered for numerical constructing the level sets of the value function (the solvability sets) under rather general description of the objects' linear dynamics. Numerical examples of this constructing are given. For the case of "strong" pursuers, the optimal feedback controls are found. For other cases (two "weak" pursuers, one strong and one weak pursuers, etc.), the problem of effective constructing players' strategies seems to be more difficult.

\section{ACKNOWLEDGEMENTS}

We are heartily grateful to the reviewers for very useful comments and recommendations.
This work is supported by the Russian Foundation for Basic Research (projects nos. 12-01-00537 and 13-01-96055), by the Program "Dynamic systems and control theory" of the Presidium of the RAS (project no.12-П-1-1002). of the Russian Federation 02.A03.21.0006.

\section{REFERENCES}

Bryson, A.E. and Ho, Y.C. (1975). Applied Optimal Control. Optimization, Estimation and Control. Hemisphere publishing corporation, John Wiley and Sons.

Ganebny, S.A., Kumkov, S.S., Le Ménec, S., and Patsko, V.S. (2012a). Model problem in a line with two pursuers and one evader. Dyn. Games Appl., 2, 228-257.

Ganebny, S.A., Kumkov, S.S., Le Ménec, S., and Patsko, V.S. (2012b). Study of linear game with two pursuers and one evader: Different strength of pursuers. In P. Cardaliaguet and R. Cressman (eds.), Annals of the International Society of Dynamic Games, Vol. 12, 269292. Birkhauser, Boston.

Isakova, E.A., Logunova, G.V., and Patsko, V.S. (1984). Computation of stable bridges for linear differential games with fixed time of termination. In A.I. Subbotin and V.S. Patsko (eds.), Algorithms and Programs for Solving Linear Differential Games, 127-158. Institute of Mathematics and Mechanics, Ural Scientific Center, Academy of Sciences of USSR, Sverdlovsk. (in Russian).

Krasovskii, N.N. and Subbotin, A.I. (1974). Positional Differential Games. Nauka, Moscow. (in Russian).

Krasovskii, N.N. and Subbotin, A.I. (1988). Game-Theoretical Control Problems. Springer-Verlag, New York.

Kumkov, S.S., Le Menec, S., and Patsko, V.S. (2013). Model formulation of pursuit problem with two pursuers and one evader. In Advances in Aerospace Guidance, Navigation and Control, 121-137. Springer, Berlin, Heidelberg.

Kumkov, S.S., Patsko, V.S., and Shinar, J. (2005). On level sets with "narrow" throats in linear differential games. International Game Theory Review, 7(3), 285-312.

Le Ménec, S. (2011). Linear differential game with two pursuers and one evader. In M. Breton and K. Szajowski (eds.), Annals of the International Society of Dynamic Games, Vol. 11, 209-226. Birkhauser, Boston.

Patsko, V.S. and Turova, V.L. (2001). Level sets of the value function in differential games with the homicidal chauffeur dynamics. International Game Theory Review, 3(1), 67-112.

Shima, T. (2005). Capture conditions in a pursuit-evasion game between players with biproper dynamics. JOTA, 126(3), 503-528.

Shima, T. and Shinar, J. (2002). Time-varying linear pursuit-evasion game models with bounded controls. J. Guid. Contr. Dyn., 25(3), 425-432.

Shinar, J., Glizer, V.Y., and Turetsky, V. (2013). The effect of pursuer dynamics on the value of linear pursuitevasion games with bounded controls. In V. Krivan and G. Zaccour (eds.), Annals of the International Society of Dynamic Games, Vol. 13, 313-350. Birkhauser, Boston.

Shinar, J., Medinah, M., and Biton, M. (1984). Singular surfaces in a linear pursuit-evasion game with elliptical vectograms. JOTA, 43(3), 431-456. 